\title{
BMJ Global Health Towards universal health coverage: advancing the development and use of traditional medicines in Africa
}

To cite: Kasilo OMJ, Wambebe C, Nikiema J-B, et al. Towards universal health coverage: advancing the development and use of traditional medicines in Africa. BMJ Global Health 2019:4:e001517. doi:10.1136/ bmjgh-2019-001517

Handling editor Seye Abimbola

Received 17 February 2019 Revised 6 August 2019 Accepted 10 August 2019
Check for updates

(C) Author(s) (or their employer(s)) 2019. Re-use permitted under CC BY-NC. No commercial re-use. See rights and permissions. Published by BMJ.

${ }^{1}$ WHO Regional office for Africa, Health systems and services cluster, Brazzaville, Republic of Congo

${ }^{2}$ Tshwane University of Technology, Pretoria, South Africa

${ }^{3}$ World Health Organization, Inter-Country Support Team for Eastern \& Southern Africa, Health systems and services cluster, Causeway, Harare, Zimbabwe

Correspondence to

Dr Ossy Muganga Julius Kasilo; kasiloo@who.int

\section{ABSTRACT}

African traditional medicine (ATM) and traditional health practitioners (THPs) could make significant contributions to the attainment of universal health coverage (UHC). Consequently, the WHO provided technical tools to assist African countries to develop ATM as a significant component of healthcare. Many African countries adopted the WHO tools after appropriate modifications to advance research and development (R\&D) of ATM. An analysis of the extent of this development was undertaken through a survey of 47 countries in the WHO African region. Results show impressive advances in R\&D of ATM, the collaboration between THP and conventional health practitioners, quality assurance as well as regulation, registration and THP integration into the national health systems. We highlight the various ways investment in the R\&D of ATM can impact on policy, practice and the three themes of UHC. We underscore the need for frameworks for fair and equitable sharing of all benefits arising from the R\&D of ATM products involving all the stakeholders. We argue for further investment in ATM as a complement to conventional medicine to promote attainment of the objectives of UHC.

\section{INTRODUCTION}

African countries have embraced the sustainable development goals (SDGs) and of specific reference to health is SDG3-good health and well-being - which has as one of its 13 targets: to achieve universal health coverage (UHC). UHC is defined as ensuring that all people have access to needed health services of sufficient quality to be effective, while ensuring that the use of these services does not expose the user to financial hardship. ${ }^{1}$ The current use of African traditional medicines (ATMs) and associated expenditures in seeking care from traditional health practitioners (THPs) implores us to analyse the role of ATM in contributing towards UHC in African countries. UHC and SDG-3 can only be achieved with a stronger emphasis on primary healthcare (PHC). ${ }^{2}$ Since over $60 \%$ of people in sub-Saharan Africa (SSA) live in rural areas where conventional healthcare is

\section{Summary box}

African traditional medicine (ATM) is an important part of African culture that is recognised and accepted by Africans, despite conventional health practice.

- Scientific validation of the safety and efficacy of plant extracts derived from African medicinal plants based on African Indigenous Medical Knowledge has been facilitated by the use of WHO technical support tools.

- Investment in the continued development of ATM will accelerate the realisation of universal health coverage.

scarce, exploring the role of ATM to achieve the goals of UHC becomes important. ${ }^{3}$ Given the economic reality and cultural beliefs, empowering THPs will enable more people to access quality healthcare, which is a critical component of UHC. ${ }^{3}$

The first definition of traditional medicine in 1976 by the WHO Regional Office for Africa Expert Committee ${ }^{4}$ was later revised by WHO as: 'the sum total of the knowledge, skills, and practices based on the theories, beliefs, and experiences indigenous to different cultures, whether explicable or not, used in the maintenance of health as well as in the prevention, diagnosis, improvement or treatment of physical and mental illnesses'. Interestingly, ATM evolved and remains resilient in spite of the much more standardised western medicine. The introduction of conventional medicine led to systematic neglect of ATM, which adversely retarded its development. The neglect is partially attributed to the laws prohibiting the practice of ATM by the colonialists, lack of documentation and the assimilation of the western lifestyle by younger generations. ${ }^{5}$ However, ATM was still practised especially in rural communities where it formed the main PHC service for the people. Despite limited investment in the development of ATM, its usage for the prevention and treatment of diseases 
continue to be widespread across Africa. The reasons for the continued patronage of ATM include perceived low cost, alignment with sociocultural, religious and spiritual values and dissatisfaction with conventional medicines. ${ }^{6}$ The components of ATM practice include birth delivery, bone setting, internal medicine, child health, mental care and so on. Herbal medicines using various parts of plants constitute about $80 \%$ of ATM products. ${ }^{7}$ However, water, oils, minerals and animal parts are used alone or combined with plant parts as recipes for treating various diseases.

Generally, ATM is regarded as more accessible, affordable and acceptable to local populations and can therefore contribute to the attainment of UHC. For instance, the average ratios of THPs and medical doctors per population in SSA are respectively 1:500 and 1:40 $000 .^{8}$ Evidence shows significant use of ATM as high as $88 \%$ of respondents in a Zambian study expressing preference to visit a traditional healer when sick, ${ }^{9}$ for patients with AIDS symptoms in Malawi, ${ }^{10}$ South Africa, ${ }^{11}$ Uganda $^{12}$ and Zimbabwe ${ }^{13}$ and as the main source of healthcare for mental illnesses in Uganda. ${ }^{14}$ Due to the high proportion of patients using herbal medicines (70\% in Ghana), some health facilities have initiated the use of herbal medicines as a component of healthcare delivery. ${ }^{15}$ Significant out-of-pocket health expenditure on THP has been reported in the literature. For example, a study in South Africa reported that out-of-pocket health expenditure on THP accounted for $10 \%$ of monthly expenditures among three-quarter of the poorest quantile. ${ }^{16}$

Recognising that Africans continue to patronise THPs and use ATMs, the WHO developed a set of tools and guidelines to support the scientific development of ATMs through the identification of their medicinal components and standardisation of procedures for their use. ${ }^{17-21}$ The WHO African Regional Strategy on 'Enhancing the Role of Traditional Medicine in Health Systems ${ }^{22}$ provided the impetus and direction vis-à-vis promotion of ATM.

In this article, we report the regional status of ATMs and THPs using information from the survey conducted in 47 countries in SSA. The survey focused on the nature and level of collaboration between THPs and CHPs concerning research, training, treatment and care. The questionnaire was completed by the official in the ountry's Ministry of Health or its equivalent responsible for traditional medical practice. Besides, research institutions dedicated to traditional medicine (TM), programme managers and directors of traditional medicine departments or similar offices or agencies also completed the questionnaires.

The completed questionnaires were returned to the WHO Country Offices where a designated official for the survey reviewed the responses for validation, inconsistencies to ensure coherence and reliability of the information received through the confirmation of claims from multiple sources. A further review was done at WHO Regional Office for completeness and accuracy before analysis. The final version was then returned to the countries for validity. Information from WHO forums that reviewed progress in ATM in SSA were considered in the analysis. ${ }^{23-28}$

\section{PROGRESS IN THE DEVELOPMENT OF ATM AND PRACTICE}

The period 2000-2018 registered significant progress regarding integration of ATMs and THPs into the national health systems. table 1 shows the specific actions taken by countries in the WHO African Region (WHO-AFRO). The information in table 1 has been compiled from a baseline and subsequent surveys carried out by WHO-AFRO during 1999/2000 and 2002, 2005, 2010, 2012, 2016 and 2018. Among others, policies and legal frameworks for traditional medicine practice are in place in 40 and 38 countries, respectively, while 38 countries established national programmes in ministries of health dedicated to the coordination of traditional medicine activities. National Expert Committees on ATM were constituted in 34 countries. In addition, African institutions made progress in: (A) research and evaluation of TMs, (B) product development, (C) collaboration between THP and CHP, (D) quality assurance, regulation and registration and (E) THP integration into the national health system that are further elaborated below.

Availability of essential medicines and technologies (medical products) to diagnose and treat health problems; a capacitated, well-trained and motivated health workers to provide the services that meet patients' needs; regulatory systems strengthening for ensuring quality assurance of phytomedicines and services; functional referral systems of patients from THPs to CHPs and vice versa; provision of essential services that are person centred, effective and responsive to expectations; and economic development and selection of essential phytomedicines for integration into national health systems are among the several requirements for a country to achieve UHC. In the UHC context, research and development (R\&D) focus on safety, efficacy, quality, strength, reliability and durability of the medical products. Regulatory systems strengthening of phytomedicines has enabled an appropriate national drug regulatory authority (NDRA) to provide an independent review of both the preclinical and clinical data with emphasis on safety, efficacy and quality of the medical product. Registration of new medical products and devices is a mandatory requirement before the medical product becomes accessible to the public. R\&D of ATM leading to standardised and quality-assured phytomedicines impacts positively on the three themes of UHC. Access to phytomedicines in poor communities and villages promotes equitable service delivery. The NDRA evaluates both the preclinical and clinical data relating to the phytomedicine to ensure its quality, safety and efficacy before it can be registered. Enhanced referral systems of patients from THPs to CHPs and vice versa resulting from better communication and collaboration of these practitioners also contributes to improving access to essential health services. 


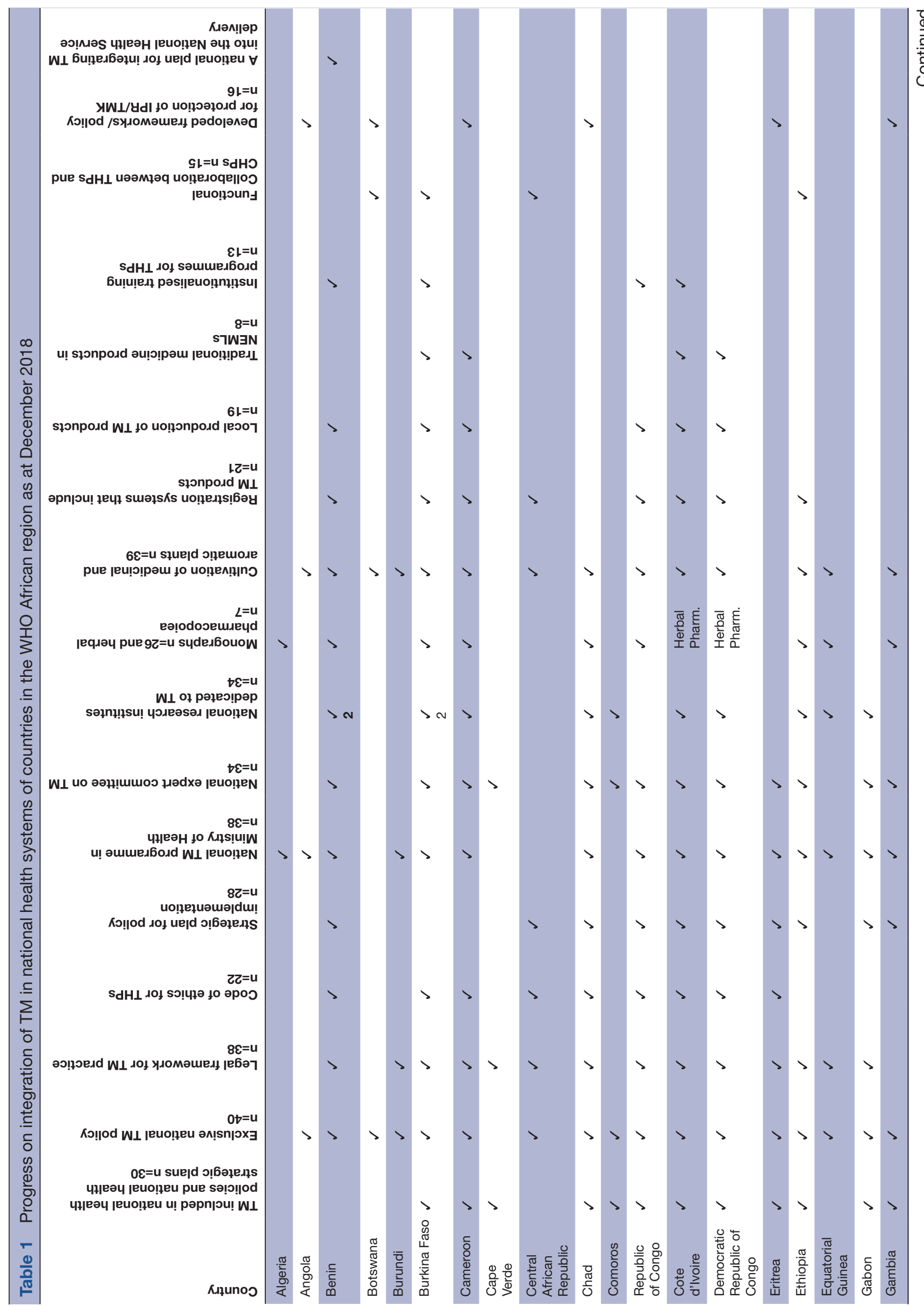




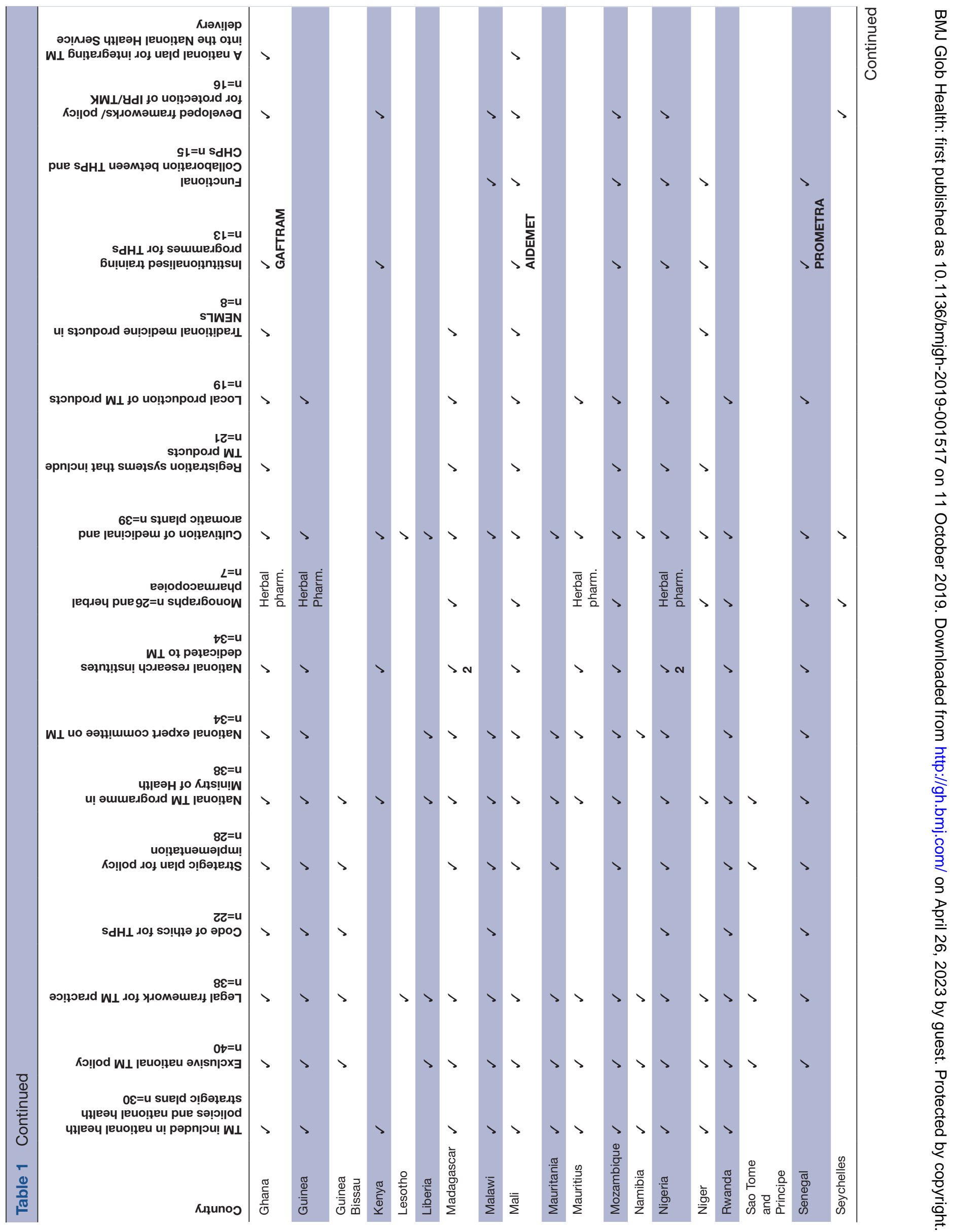




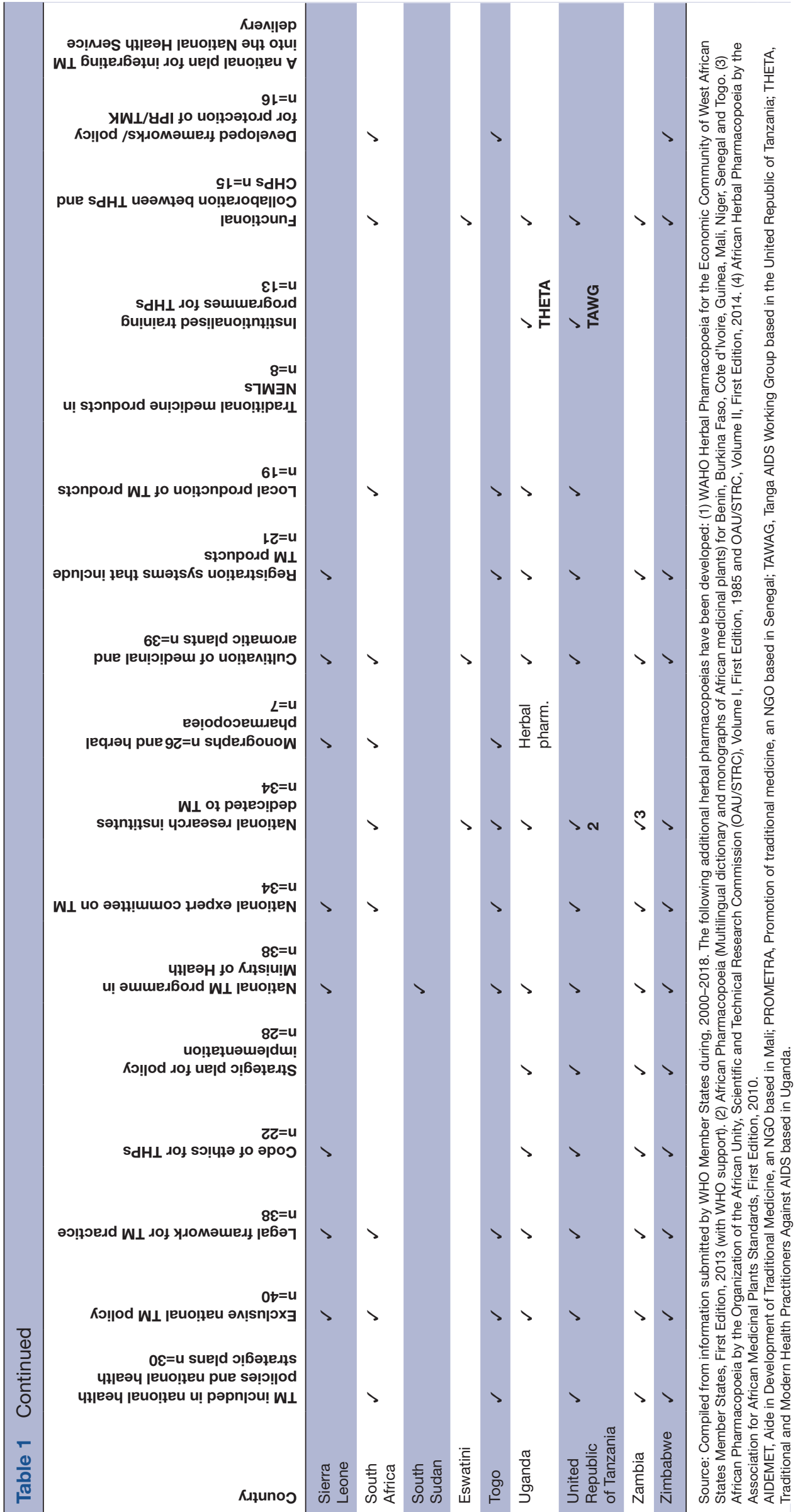


The UHC theme of quality of services, among others, includes quality of drugs and essential health services. The registration of the phytomedicine by the NDRA guarantees its quality. Well-trained, accredited or licenced THPs working as part of PHC teams increases the availability of more human resources and contributes to the provision of quality essential services that are person centred, effective and responsive to expectations given the holistic approach of traditional medicine. In addition, creation of awareness and monitoring of appropriate use of phytomedicines during targeted days such as national traditional medicine week or the African Traditional Medicine Day commemorated on 31 August of every year in Africa contribute to patient safety.

The third theme of UHC is people protection against financial risk, which is particularly important for the populations living below or close to poverty who can be pushed into catastrophic situations because of health expenses. The affordability of improved herbal medicines to reduce out-of-pocket health expenditures is one of the strong pillars of ATM. For example, in Ghana, the government reimburses patients' consultations with THPs through the insurance scheme and is planning to do the same for the phytomedicines included in its national essential medicines lists. In some cases, such as in Nigeria, after the herbal medicine for treating sickle cell disease (SCD) was standardised for the THP by NIPRD, he continued to give his patients the improved herbal medicine without any charge. Economic development emanating from the commercialisation of medicinal and aromatic plants and phytomedicines also contributes to achieving UHC. However, appropriate health expenditures need to be made in a manner that protects people against financial risk for movement towards UHC.

Since the target of UHC is quality health services for all, a collaboration between well-trained, accredited THP and CHP is crucial to attaining such a goal. Such collaboration promotes knowledge and skills of the THP, especially in basic hygiene, proper diagnosis, health promotion, monitoring, mobilisation for immunisation and so on. The services by trained THPs in rural communities where western trained health personnel are not available promotes two of the themes of UHC namely, equity to health services and quality of health services. Integration of THP into the national health services will guarantee improved services coverage and health outcomes and recognition of their services. Also, THPs can practice freely under appropriate government regulations to reach people who believe in ATM and cannot afford health costs in conventional health facilities. Therefore, investing in THP's integration into PHC, documenting ATM and reporting regularly as part of a well-functioning health information system is the most cost-effective way to ensure access and quality to essential healthcare, which will significantly contribute towards the achievement of UHC.

Specifically, integrating ATM into national health systems including certification of THPs will drastically reduce the fake THPs who only practice for financial benefits with serious adverse effects on their ignorant patients. Consequently, the integration of ATM into national health services directly promotes all the three themes of UHC. Equity to health services is promoted by the availability of trained licensed THPs to provide their expertise to the communities. Quality of health services is guaranteed by training, retraining and monitoring of the THPs. Protection of the population against financial risks is promoted by access to improved herbal medicines that can effectively replace the fake products marketed in poor communities.

\section{RESEARCH AND DEVELOPMENT}

Research into ATM drew the interest of at least 34 research institutions in 26 African countries (table 1) by 2018 as compared with 18 and 20 in 2000 and 2012, respectively. These institutes researched the use of ATM products for priority diseases such as HIV/AIDS, SCD, diabetes and hypertension using WHO guidelines. ${ }^{29} \mathrm{~A}$ total of 14 countries used research results to authorise the marketing of certain traditional medicine products used for communicable and non-communicable diseases, while eight countries included traditional medicine products in their national essential medicines lists (table 1).

The establishment in 1971 of the International Association for the Promotion of Traditional Medicine (PROMETRA), a non-governmental organisation, in Senegal is one of the earliest efforts to promote the standardisation and use of ATMs in healthcare delivery. PROMETRA conducts scientific research, hosts international conferences and cultural exchanges, publishes a quarterly bilingual journal, Medicine Verte, ${ }^{29}$ and coordinates a diaspora-wide network on TM in partnership with academic institutions throughout Africa, for example, Noguchi Research Institute of the University of Ghana and University of Venda in South Africa, USA, (eg, Morehouse School of Medicine, Atlanta, and North Caroline Central University, BRITE Institute) among others. PROMETRA subsequently established the Centre for Experimental Traditional Medicine in Fatick, Senegal, to conduct TM research and foster THP-CHP collaboration with respect to patient management, treatment and research. This effort was accorded recognition by the government and facilitated THP-CHP collaboration in Senegal. ${ }^{30}$

In Ghana, R\&D of herbal products for the management of various diseases is undertaken by the government-owned Centre for Scientific Research into Plant Medicine (CSRPM), which boasts of a long-standing collaboration with THPs since 1975. The CSRPM routinely identifies and consults with THPs to diagnose, prescribe, administer and monitor the effects of selected herbal products on outpatients and has at least 35 state-registered products for the management of various diseases. $^{3132}$ 
In a bold initiative, the Faculty of Pharmacy, Obafemi Awolowo University in Nigeria, took the unusual step of appointing, in 1972, a THP as a full-time faculty member with responsibility for training and research in TM, thus taking the THP to the corridors of western apex learning and intellectual discourse and becoming the first consultant herbalist in Africa. The success of that action led to the recruitment of other THPs to enrich the training of pharmacy students and to advance research into ATM using the unwritten knowledge and skills of THPs. The establishment of botanical gardens for growing herbal plants and raising other TM raw materials increased the profile of the institution rather than diminish it as one would have expected given the official disdain for TM by western educated intellectuals who viewed the use of herbs as fetish at best and evil in its worst. Other institutions and individuals were emboldened to seek grants and carry out research into indigenous health practices. The THPs roles were not limited to the collection of recipes. They were involved in establishing validity, evaluation and verification of claims made on medicinal plants by other practitioners. This approach is recommended for the training of pharmacists in Africa, demystifying and removing the confidentiality surrounding the knowledge transfer about TMs and THPs. The faculty membership was mutually beneficial to the THP and the research institution. The THP acquired research skills, contributed to several research initiatives and conferences on TM and its practice and was recognised in the university for outstanding contribution to knowledge including the documentation of the ethnobotanical flora of Western Nigeria. 3334

Mali is the first country to establish a state-owned research institute devoted to investigating TM in Africa. Initially referred to as National Institute of Phytotherapy and TM, in 1968, the name changed to the National Institute for Research on Public Health (NIRPH). The institute provides evidence regarding the safety and efficacy of TM before it can be awarded a marketing authorisation.

Although many African countries including Uganda, Ethiopia, Kenya and Tanzania have evaluated some aspects of traditional health practices, Burkina Faso presents a comprehensive example of how countries have attempted to evaluate THPs. Burkina Faso has a Centre for Experimental Research, which took on the evaluation of TMs that THPs use for the management of opportunistic infections related to AIDS, malaria, SCD and dracunculiasis. A classic example was the screening of 25000 school children for SCD and the application of extracts from five plants that the THPs had claimed as efficacious for SCD management using in vitro evaluation of their effect. The study concluded that one of the plants, a wellknown traditional medicinal herb, provided scientific evidence as safe, efficacious and effective in the management of SCD. ${ }^{35}$ This approach was modified to evaluate the efficacy of plant extracts claimed to have effect on the management of opportunistic infections related to AIDS. A multidisciplinary team that monitored the patients observed a significant decline in the viral load in the patients. ${ }^{36}$ In the study, the medical doctor managed opportunistic infections, the pharmacognosy laboratory of the University of Ouagadougou formulated tablets and was responsible for the quality control of TMs. Burkina Faso invested in evaluation of other medicinal plants for their proven pharmacological effects. Burkina Faso evaluation demonstrated that traditional herbal medicines had clinical benefit for the management of people living with HIV/AIDS who were not at the terminal stage. The various collaborative arrangements with THPs strengthened their skills, and facilitated their recognition vis-à-vis their indigenous medical knowledge and unique services in poor communities.

\section{PRODUCT DEVELOPMENT}

SCD is a major public health problem in SSA. In many African countries, $10 \%-40 \%$ of the population carries the sickle cell gene resulting in an estimated SCD prevalence of at least $2 \% .{ }^{37}$ It is not surprising that THPs have the knowledge and skill for managing SCD. The National Institute for Pharmaceutical Research and Development (NIPRD) in Abuja evaluated the various extracts obtained from medicinal plants for the prophylactic management of SCD in collaboration with the THP that released the recipe to NIPRD.$^{38}$ The pilot clinical study showed $73 \%$ effectiveness at managing SCD crisis and the remaining had infrequent but less severe crisis over 12 months of medicine use. A randomised placebo-controlled double-blind crossover clinical trial that lasted for 12 months showed that the ATM product, NIPRISAN, significantly reduced the frequency of SCD crisis associated with severe pains. Liver and renal functions were normal thus confirming that NIPRISAN is a safe and efficacious phytomedicine for the management of patients with SCD. ${ }^{38}$ The Nigerian government subsequently licenced the commercial production and global marketing of NIPRISAN. The product is patented in the USA and 46 other countries.

The Mali research institute carried out a largescale screening of 66 plants that are in use by THPs for malaria treatment and subjected the extracts to in vitro and in vivo tests. Extracts of the plant, Argemone (A). mexicana indicated significant efficacy in both in vitro and in vivo testing ${ }^{39}$ subsequently subjected it to a randomised control clinical study. With A. mexicana decoction, there was complete malaria parasite clearance on the seventh day, down from 35000 per $\mu \mathrm{L}$ and costs only $5 \%$ the price of artesunate combination therapies. Madagascar has a patent for a medicinal product used for the treatment of uncomplicated diabetes (type II) ${ }^{40}$ Similarly, Uganda has patented at least five TM products, two of which are used for the treatment of malaria and relief of benign prostate hypertrophy, whereas the United Republic of Tanzania has filed two patents, being an invention for a herbal juice for use as a nutritional supplement and a pharmaceutical 
product based on medicinal plants used for treatment of skin fungal infections and allergic skin conditions. ${ }^{41}$

In Zimbabwe, research into ATM for the management of HIV/AIDS was carried out at the University of Zimbabwe leading to the production of the herbal medicine, gundamiti, with up to $97 \%$ reduction of the viral load in patients with HIV/AIDS. Gundamiti also had an appreciable effect on the decrease in HIV/ AIDS-related opportunistic infections without affecting kidney and liver function. ${ }^{42}$ An interesting finding is that several countries have patented their TM products, while others were registered by national regulatory authorities. These new phytomedicines have been added to the global arsenal of medicines for serious disorders. These new medical products promote the quality of health services available to poor communities who may not be able to travel to cities for western health services.

\section{COLLABORATION BETWEEN THP AND CHP}

Fostering collaboration between THPs and CHPs, who often disregard the contribution of THPs to healthcare delivery, is one of the main challenges. However, this situation is changing in many African countries where stakeholders had fostered cooperation between THP and CHP. For example, in South Africa, Rachel et at $t^{43}$ reported evidence-based recommendations to facilitate professional collaboration between CHPs and THPs. Similarly, in Mali, the Department of Traditional Medicine invests in the education of THPs through regular joint capacity building and knowledge sharing workshops for both THPs and CHPs. Such avenues provide opportunities for understanding across practice orientations, facilitate collaborative exchanges on the classification of various diseases, diagnosis, treatment and management procedures.

Another example is Senegal where patients are referred to both THPs and CHPs. The CHPs monitor the patient taking vital signs, diagnosing and analysing laboratory tests before the THP treats the patient within the health facility. The patient is observed at the conventional medical unit for the impact of traditional medical treatment using pretreatment and post-treatment comparisons. The collaboration has promoted mutual respect for both cadre of health practitioners, reduced health workers' scepticism and increased appreciation and the development of ATM. This approach to collaboration has also been demonstrated to some extent in Uganda, ${ }^{44}$ Tanzania ${ }^{45}{ }^{46}$ and South Africa, ${ }^{47}$ concerning HIV/AIDS counselling and behavioural change.

Collaborative HIV/AIDS, sexually transmitted infections (STI) and tuberculosis (TB) programmes involving THPs have been initiated in several SSA countries ${ }^{41} 48$ with varying degrees of success. In South Africa's Kwazulu Natal province, THPs were trained to assist in practice-related risk reduction techniques and to educate, refer and follow-up patients under STI/ HIV and TB care. ${ }^{49}$ Despite its effectiveness, THP-CHP collaboration in Eastern and Southern African countries have been met with more caution than in Western Africa. Different countries demonstrate that collaboration between THPs and CHPs can occur in various ways but invariably should result in cohesive teams that are willing to harness each other's potentials for the benefit of the patient, the community and the wider society. When both categories of practitioners collaborate, they can enhance practices within the healthcare system, manage collaborative clinics, undertake collaborative research and continue the development of new medicines.

The collaboration between THPs and CHPs promote access to health services by rural dwellers where most of the THPs practice. The THP-CHP collaboration involves, among others, training of THPs in PHC and early signs for referrals of their patients. Similarly, the CHPs become conversant with ATM pathophysiology of diseases and diagnostic techniques and can then engage in case studies with them. Consequently, such a practice promotes quality of health services by both THPs and CHPs, which aligns with one of the themes of UHC.

\section{THP PROFICIENCY EVALUATION, REGISTRATION AND INTEGRATION INTO THE HEALTH SYSTEM}

Mali introduced several complementary structural and functional approaches for evaluating the competency of THPs, registering them and integrating them into the healthcare delivery system. The TM department registers THPs in coordination with the NIRPH, Bamako. The department certifies and registers THPs who meet stipulated criteria. ${ }^{50}$ One crucial criterion is the evaluation by the district CHP of the individual's practice involving 30 cases per disease regarding efficacy and safety for about 4 months. The CHP prepares a report, which is sent to Bamako. Any THP who is not registered is regarded as a quack. The registration criteria that combine scientifically proven evidence of professional competence, local acknowledgement and recognition as well as acceptance by the CHP counterpart are worthy of consideration for adoption by other countries.

Irrespective of the country's practices, majority of THPs who collaborated with CHPs had been accredited to practice. Mali represents a very vibrant traditional health practice in SSA with 51 THP associations, a national bureau and a THP federation. The association in collaboration with government hosts the annual regional International African Traditional Medicine Week. ${ }^{51}$ These efforts enhance the integration of THP into PHC and thus accelerate the attainment of UHC. The registration system for THPs is crucial to reducing the widespread sale of fake herbal drugs in villages and rural communities with unsubstantiated claims because 
only certified THPs are permitted to market their products. As we highlight the role of ATM in UHC, we note that it is not without challenges. The range of services provided by THPs, the costs involved and the quality of their services need to be addressed continually.

\section{IMPLICATIONS FOR POLICY AND PRACTICE}

A critical need is financial and human resources to develop standardised phytomedicines for multicentre randomised controlled clinical trials after establishing safety, efficacy and quality of the potential products at preclinical levels. Such wise investments will impact on both policy and practice in specific ways. For example, the policy on functional integration of ATM into national health systems and professional collaboration between THPs and CHPs will be enhanced. The functional integration of ATM into the national health systems will encourage genuine THPs to collaborate with the government leading to technical and financial support for the THP. Subsequently, the infrastructure and capacity to produce herbal medicines are greatly enhanced, while coverage for the public use of the improved herbal medicines is enlarged. Such a trend directly promotes the three themes of UHC. Equity in health services is enhanced when improved herbal medicines are available to a large proportion of the population. Since the quality of the improved herbal medicines must first be certified by the NDRA before their registration, their quality is guaranteed. Furthermore, people protection against financial risks is promoted by the affordability of improved herbal medicines. Intellectual property (IP) protection of innovations of commercial application arising from African Indigenous Medical Knowledge (AIMK) may be challenging using the normal patent protocols. In many cases, the AIMK is held by the entire community. Sometimes, AIMK is held by communities in different countries since biodiversity and cultural affinities defy the artificial borders created by the colonialists. In such cases, sui generis (of its own kind) can be adopted by the country to protect their IMK. Intellectual property protection is crucial to the preservation of AIMK and African medicinal plants and, subsequently, their continued therapeutic applications. Once the medicinal values of the plants are recognised, the national authorities will conserve such plants from deforestation, industrial developments, para cities and so on. The private sector will cultivate such medicinal plants. Thus, without IP protection of AIMK, the positive impacts on the three themes of the UHC will be hampered due to non-availability of the improved herbal medicines.

It is noteworthy that national policies on commercialisation of African phytomedicines with proven safety and efficacy and their global marketing will subsequently generate jobs for young Africans, promote socioeconomic status of communities (especially women) where the medicinal plants will be cultivated and harvested and impart new technical skills to Africans in agronomy and the pharmaceutical sector. The practical outcomes of such a trend is the enhanced quality of life of Africans with evidence in the reduction of communicable and non-communicable diseases, poverty alleviation, increased lifespan and so on. It is well established that investment in health yields profitable returns. Consequently, African countries that adopt these recommendations will also benefit economically, socially and politically from innovations and health products based on ATM.

\section{CONCLUSION}

The significant progress made by some African countries in the development and integration of ATM and THPs into the national health systems provides lessons that can be harnessed to improve access to quality health services and subsequently contribute to the attainment of UHC. The research infrastructure and mechanisms in place provide a platform for further development of efficacious and affordable ATM. Research institutions dedicated to the R\&D of ATM, comprehensive procedures for evaluating THP claims, assessing the competencies of THPs and certifying them to practice are some of the good practices that can be scaled up. In furtherance of the contribution of ATM to UHC, establishment of Regional Centers of Excellence for R\&D possibly around regional economic communities to harness research expertise and resources; allocation of adequate funding to undertake $R \& D$ especially phase III clinical trials of safe and effective ATM products; enhanced functional collaboration between THPs and CHPs; and marketing of products with evidence on safety, efficacy and quality registered at national levels across the continent and their inclusion in national essential medicines lists should be actively promoted.

Conforming to the Nagoya Protocol, countries should develop frameworks for fair and equitable sharing of all financial and non-financial benefits arising from the R\&D of ATM products involving all the stakeholders especially individuals and communities who are the custodians of traditional medical knowledge. Such a practice will further promote collaboration between THPs and biomedical researchers thereby increasing the availability of new affordable and accessible phytomedicines for management of different diseases.

Acknowledgements We acknowledge the support received from Professor Oladele Akogun in the finalisation of this article.

Contributors OMJK: conceived and designed the analysis, developed the data collection questionnaire, led the drafting of the paper and reviewed it critically based on the reviewers' comments. CW: contributed data, performed the data analysis and contributed to writing the paper. J-BN: contributed data and its interpretation, reviewed the paper and provided valuable contribution. JN-0: substantial contributions to analysis of data, the significance of this analysis paper and its review and contributed to the drafting of the paper.

Funding The authors have not declared a specific grant for this research from any funding agency in the public, commercial or not-for-profit sectors. 
Disclaimer The inclusion of the herbal formulations in this paper should not be interpreted as an implicit endorsement of the quality, safety and efficacy of these products by the WHO but rather sharing information to stimulate research.

Competing interests None declared.

Patient consent for publication Not required.

Provenance and peer review Not commissioned; externally peer reviewed.

Data availability statement Data are available with the WHO Africa region office.

Open access This is an open access article distributed in accordance with the Creative Commons Attribution Non Commercial (CC BY-NC 4.0) license, which permits others to distribute, remix, adapt, build upon this work non-commercially, and license their derivative works on different terms, provided the original work is properly cited, appropriate credit is given, any changes made indicated, and the use is non-commercial. See: http://creativecommons.org/licenses/by-nc/4.0/.

\section{ORCID iDs}

Ossy Muganga Julius Kasilo http://orcid.org/0000-0003-1538-9293

Juliet Nabyonga-Orem http://orcid.org/0000-0002-1061-8678

\section{REFERENCES}

1 World Health Organisation. World health report. Geneva Switzerland, 2010.

2 World Health Organization, United Nations Children's Fund (UNICEF). From Alma-Ata470 towards universal health coverage and the sustainable development goals. Global Conference on Primary Health Care Astana- Kazakhstan, 2018.

3 Growing G. The majority of sub-Saharan Africans live in rural areas, 2014. Available: www.global-growing.org/en/content/fact-1-majoritysub-saharan-africans-line-rural-areas-european-predorminantlycities

4 World Health Organization. Traditional, complementary and integrative medicine 2019 [Definitions. Brazzaville Congo, 2019. https://www.who.int/traditional-complementary-integrativemedicine/about/en/

5 Parliament of South Africa. Witchcraft suppression act 3 of 1957 , 1957. https://en.wikisource.org/wiki/Witchcraft_Suppression_Act 1957

6 James PB, Wardle J, Steel A, et al. Traditional, complementary and alternative medicine use in sub-Saharan Africa: a systematic review. BMJ Glob Health 2018;3.

7 Mahomoodally MF. Traditional medicines in Africa: an appraisal of ten potent African medicinal plants. Evid Based Complement Alternat Med 2013;2013:1-14.

8 Zhang Q. Global situation and who strategy on traditional medicine. Traditional Medicine and Modern Medicine 2018;01:11-13.

9 Stekelenburg J, Jager BE, Kolk PR, et al. Health care seeking behaviour and utilisation of traditional healers in Kalabo, Zambia. Health Policy 2005;71:67-81.

10 Hatchett LA, Kaponda CPN, Chihana CN, et al. Health-seeking patterns for AIDS in Malawi. AIDS Care 2004;16:827-33.

11 Nlooto M, Panjasaram Naidoo P. Clinical relevance and use of traditional, complementary and alternative medicines for the management of HIV infection in local African communities, 19892014: a review of selected literature. Journal of African Studies 2014;28.

12 Asiimwe S. Nutri-medicinal plants used in the management of HIV/ AIDS opportunistic infections in Western Uganda: documentation, phytochemistry and bioactivity evaluation. doctoral thesis, 2015. Available: https://www.diva- portal.org/smash/get/diva2./ FULLTEXT02.pdf

13 Mandizadza E, Chavunduka G, Chapter S. Traditional medicine and HIV and AIDS treatment: challenges, prospects and lessons for Zimbabwe. In: Tizazu G, Mesfin R, eds. Antiretroviral treatment in sub-Saharan Africa: challenges and prospects. ed. Organisation for social science research in eastern and southern Africa, 2015.

14 Nsereko JR, Kizza D, Kigozi F, et al. Stakeholder's perceptions of help-seeking behaviour among people with mental health problems in Uganda. Int J Ment Health Syst 2011;5:5.

15 Boateng MA, Danso-Appiah A, Turkson BK, et al. Integrating biomedical and herbal medicine in Ghana - experiences from the Kumasi South Hospital: a qualitative study. BMC Complement Altern Med 2016;16:189.

16 Nxumalo $\mathrm{N}$, Alaba O, Harris B, et al. Utilization of traditional healers in South Africa and costs to patients: findings from a national household survey. J Public Health Policy 2011;32 (Suppl 1):S124-S136.
17 WHO regional office of Africa. Guidelines for clinical study of traditional medicines in the WHO African region. Brazzaville Congo, 2004

18 World Health Organization, WHO Technical Report Series. Guidelines for good clinical practice (GCP) for trials on pharmaceutical products. Geneva Switzerland, 1995.

19 WHO Regional Office for Africa. Guidelines on registration of traditional medicines in the WHO African region. Brazzaville Congo, 2004.

20 World Health Organization. Training in tropical diseases. In: Good laboratory practice training manual: trainer. Switzerland Geneva, 2008.

21 World Health Organization. WHO Guidelines on Good Agricultural and Collection Practicesfor medicinal plants. Geneva Switzerland, 2003.

22 WHO Regional Office for Africa. Regional Committee for Africa, enhancing the role oftraditional medicine in health systems: a strategy for the African region (Document AFR/RC63/6). In: . Brazzaville Congo, 2013.

23 UNICEF/UNDP/World Bank/WHO Special Programme for Research and Training inTropical Diseases (TDR). A preparatory group meeting, Johannesburg 27-28 June,2002. Handbook on Non-Clinical Safety Testing. Geneva Switzerland, 2004.

24 WHO Regional office for Afica. Final report of the regional workshop on development of country work plans for implementation of the regional strategy on enhancing the role of traditional medicine in health systems (Document AFR/EDM/TRM/2015.1). Zimbabwe: Harare, 2015

25 WHO Regional Office for Africa. Pre-Clinical safety testing of traditional medicines. South Africa: Johannesburg, 2004.

26 WHO Regional Office for Africa. Final report of the consultative meeting on pre-clinical safety testing of traditional medicines. Brazzaville Congo, 2005.

27 WHO Regional Office for Africa. Final report of the regional workshop on research and development of traditional medicines. Zimbabwe: Harare, 2012.

28 WHO Regional Office for Africa. Final report of regional workshop on research and development of traditional medicine and intellectual property rights (Document AFR/TRM/04.2). South Africa: Johannesburg, 2004

29 PROMETRA International. Medecine Verte. Science and writing for an African renaissance. Scientific Research and Information Journal 2016.

30 Busia K, Kasilo OMJ. Collaboration between traditional health practitioners and conventional health practitioners: some country experiences. African Health Monitor 2010;13:40-6.

31 Asiedu - Larbi J, Adjimani JP, Okine LKN, et al. Efficacy studies on Mist Diodia, a herbal preparation for the management of hypertension in rodents. Med Aromatic Plants Res J 2014;2:6-7.

32 Thomford KP, Edoh DA, Thomford AK, et al. Effectiveness of the combination of Cryptolepis sanguinolenta and Clausena anisata in uncomplicated malaria. Int $\mathrm{J}$ Chem and Pharm Sci 2014;2:1367-70.

33 Awoyemi OK, Ewa EE, Abdulkarim I ea. Ethnobotanical assessment of herbal plants in south western Nigeria. Acad Res Int 2012;2:50-7.

34 Soladoye MO, Amusa NR, ea aji-ESO. Ethnobotanical survey of anticancer plants in Ogun state, Nigeria. Annals of Biological Research 2010;1:261-73

35 Nikiema JB, Ouattara B, Sembde R, et al. Promotion de la médecine traditionelle du Burkina Faso:essai de développement d'un médicament antidrépanocytaire, le Faca. African Health Monitor Special Issue on Decade of African Trdaitional Medicine,(2001-2010) 2010;14:52-7.

36 Simpore J, Nikiema JB, Sia D. Evaluation of traditional medicines for the management of HIV/AIDS: the experience of Burkina Faso. Traditional medicine: Our Culture OurFuture. African Health Monitor 2003:4:29-30.

37 Fleming AF, Storey J, Molineaux L, et al. Abnormal haemoglobins in the sudan savanna of Nigeria. I. prevalence of haemoglobins and relationships between sickle cell trait, malaria and survival. Ann Trop Med Parasitol 1979;73:161-72.

38 Wambebe C, Khamofu H, Momoh JA, et al. Double-Blind, placebocontrolled, randomised cross-over clinical trial of NIPRISAN in patients with sickle cell disorder. Phytomedicine 2001;8:252-61.

39 Willcox ML, Graz B, Falquet J, et al. Argemone mexicana decoction for the treatment of uncomplicated falciparum malaria. Trans $R$ Soc Trop Med Hyg 2007:101:1190-8.

40 Sharma SB, Nasir A, Prabhu KM, et al. Antihyperglycemic effect of the fruit-pulp of Eugenia jambolana in experimental diabetes mellitus. J Ethnopharmacol 2006;104:367-73. 
41 Kayombo EJ. Indigenous knowledge and HIV/AIDS prevention and management in local communities in Africa South of the Sahara. Pharmaceut Anal Acta 2013;04

42 Kasilo OMJ. Enhancing traditional medicine research and development in the African region. Traditional medicine: Our Culture Our Future. African Health Monitor 2003;1:5-18.

43 Rachel M, van Rooyen B, Nomazwi P, et al. Evidence-Based recommendations to facilitate professional collaboration between allopathic and traditional health practitioners. Health SA Gesondheid 2017;22:291-9.

44 Kyeyune P, Balaba D JH. The Role of Traditional Health Practitioners in increasing access593 to HIV/AIDS Prevention and Care: The Ugandan Experience. Traditional medicine: Our Culture, Our Future. African Health Monitor 2003;4:31-3.

45 Mtulu S. Traditional Medicine and HIVIAIDS. The training course on local health practice for MAP project team and NAC's. Tanga: Tanzania, 2005.

46 Prakash S. Tanga AIDS working group case study. Available: http:// siteresources.worldbank.org/INTINDKNOWLEDGE/Resources/Tawg.pdf
47 Green EC, Zokwe B, Dupree JD. The experience of an AIDS prevention program focused on South African traditional healers. Soc Sci Med 1995;40:503-15.

48 Colvin M, Gumede L, Grimwade K, et al. Contribution of traditional healers to a rural tuberculosis control programme in Hlabisa, South Africa. Int J Tuberc Lung Dis 2003;7-S86-91.

49 Gqaleni N, Hlongwane T, Khondo C. Biomedical and traditional healing collaboration on HIV/AIDS in KwaZulu natal, South Africa. International Journal on Human Development and International Cooperation 2014;2:1-9.

50 Diallo D, Koumare M, Traore AK. Collaboration between traditional health practitioners and conventional health practitioner: the Malian experience. African Health Monitor 2003;13.

51 Kasilo OMJ, Trapsida J-M, Mwikisa CN. An overview of the traditional medicine situation in the African region. Special Issue on Decade of African Traditional Medicine (2001-2020). African Health Monitor 2010 2010;13:7-15. 